\title{
Management of transient ischaemic attacks and stroke
}

\author{
PRD Humphrey
}

\section{Summary}

The management of stroke and transient ischaemic attacks (TIAs) has changed greatly in the last two decades. The importance of good blood pressure control is the hallmark of stroke prevention. Large multicentre trials have proven beyond doubt the value of aspirin in TIAs, warfarin in patients with atrial fibrillation and embolic cerebrovascular symptoms, and carotid endarterectomy in patients with carotid TIAs. There seems little doubt that patients managed in acute stroke units are more likely to be independent at six months than those managed in a general medical ward. This article emphasizes the importance of basing clinical management on simple history taking and examination and appropriate investigation. This, combined with knowledge of the natural history risk of TIA and stroke and the results of randomised trials, allows individuals to be managed in the most appropriate manner. This review is designed to be a practical guide, useful in the day to day management of patients with cerebrovascular disease.

Keywords: transient ischaemic attacks, stroke, management, clinical guidelines

\section{Common symptoms of carotid TIAs}

- hemiparesis - complete or partial

- hemisensory loss - complete or partial

- dysphasia

- loss of vision in one eye (amaurosis fugax)

Box 1

The Walton Centre for Neurology \& Neurosurgery, Rice Lane, Liverpool L9 1AE, UK

PRD Humphrey
The management of stroke and transient ischaemic attacks (TIAs) consumes about $5 \%$ of NHS hospital costs. Stroke is the commonest cause of severe physical disability with an annual incidence of two per $1000 .{ }^{1}$ In an 'average' district general hospital of 250000 people there will be 500 first strokes in one year with a prevalence of about 1500. TIAs are defined as acute, focal neurological symptoms, resulting from vascular disease which resolve in less than 24 hours. The incidence of a TIA is 0.5 per 1000 .

Stroke is not a diagnosis. It is merely a description of a symptom complex thought to have a vascular aetiology. It is important to classify stroke according to the anatomy of the lesion, its timing, aetiology and pathogenesis. This will help to decide the most appropriate management.

\section{Classification of stroke}

Many neurologists have described erudite vascular syndromes in the past. Most of these are of little practical use. However, a broad-based anatomical knowledge is of importance as this has significance both in terms of pathogenesis and management.

\section{ANATOMICAL}

\section{Carotid vs vertebrobasilar}

Carotid Classifying whether a stroke is carotid or vertebrobasilar in territory is important especially if the patient makes a good recovery. Carotid endarterectomy is of proven value in those with carotid symptoms. Carotid stroke usually produces hemiparesis, hemisensory loss or dysphasia. Apraxia and visuospatial problems may also occur. If there is a severe deficit, there may also be a homonymous hemianopia and gaze palsy. Episodes of amaurosis fugax or central retinal artery occlusion are also carotid events (box 1).

Vertebrobasilar The terminal branches of this system are the posterior cerebral artery, ischaemia of which usually produces unilateral visual field defects. Bilateral symptoms are not uncommon with complete blindness or bilateral visual hallucinations (eg, impression of frosted glass or water running across the whole of the vision).

The posterior cerebral artery also supplies part of the thalamus: infarction here produces sensory impairment over the contralateral side of the body. This may be accompanied by a very unpleasant pain which may be spontaneous or induced by light touch (thalamic pain) and often only reaches its peak some months after the stroke.

The brainstem signs following vertebrobasilar ischaemia depend on the level of the lesion in the brainstem. Midbrain ischaemia may result in pupillary changes with impaired vertical gaze or oculomotor nerve dysfunction. Damage to the pons produces horizontal gaze palsy with facial weakness or sensory loss. In either case a quadriparesis or hemiparesis may occur.

A wide range of other syndromes are reported to follow ischaemia of localised areas of the brain. The basic pattern is one of ipsilateral cranial nerve palsies and cerebellar disturbance combined with contralateral paresis or sensory loss which may affect face, arm and leg or just arm and leg, depending on the level in the brainstem at which this occurs. A Horner's may be seen.

The 'locked-in' syndrome is important to elicit. The patient appears unconscious but is fully conscious and can only move his eyes vertically and sometimes his eyelids. It is good practice to introduce oneself to any patient who appears to be unconscious and immediately ask the patient to move his eyes before accepting that the patient is truly unconscious.

Sometimes amnesic symptoms may be seen. However, in most patients 


\begin{tabular}{|cc|}
\hline \multicolumn{2}{|c|}{ Common lacunar infarcts } \\
\hline $\begin{array}{c}\text { Clinical type } \\
\text { pure motor } \\
\text { hemiplegia }\end{array}$ & $\begin{array}{c}\text { Site of lesion } \\
\text { internal capsule, } \\
\text { pons, cerebral } \\
\text { peduncle } \\
\text { thalamus } \\
\text { pure hemi- } \\
\text { anaesthesia }\end{array}$ \\
$\begin{array}{c}\text { ataxic hemi- } \\
\text { paresis }\end{array}$ & $\begin{array}{c}\text { pons, internal } \\
\text { capsule } \\
\text { dysarthria/clumsy } \\
\text { hand syndrome } \\
\text { pons, internal } \\
\text { capsule }\end{array}$ \\
\hline
\end{tabular}

Box 2 transient global amnesia, which lasts several hours, is no longer thought to be a TIA.

\section{Lacunar}

These small deep micro infarcts described by Fisher are commonly seen in hypertensive and diabetic patients. ${ }^{2}$ They rarely occur in patients with carotid stenosis. It is important to attempt to recognise these lacunar syndromes because of their good prognosis and different pathogenesis. Lacunar infarcts commonly present as pure motor stroke, pure sensory stroke, sensorimotor stroke or ataxic hemiparesis (box 2). Patients must have either complete face, arm and leg or major face and arm or leg involvement. Those with more restricted deficits, eg, weak hand only, are not included - these are considered to be cortical events, often embolic in aetiology. Acute focal movement disorders may also be lacunar (eg, hemiballismus).

Sometimes multiple lacunar infarcts occur. In such cases, there is often, but by no means always, a history of preceding stroke. The resulting syndrome is of a pseudobulbar palsy with dementia, dysarthria, small stepping gait (marche a petits pas) unsteadiness and incontinence.

\section{Subclavian steal syndrome}

This syndrome is largely an irrelevance. Subclavian stenosis is common in asymptomatic patients. The classical syndrome of brainstem ischaemia on exercising the arm is rarely present. It has a very low risk of stroke $(<2 \%$ per annum). It can usually be suspected by measuring the blood pressure in both arms. It is not necessary to consider any form of surgical intervention unless there are intractable vertebrobasilar TIAs. Angioplasty is a less invasive option.

\section{Border zone infarcts}

Sometimes infarction follows a generalised reduction in cerebral blood flow This is most commonly seen after a cardiac arrest or hypoxic damage during cardiac surgery. Ischaemia is then especially marked in the border zone between the territory of individual arteries because here perfusion is least. The parieto-occipital zone is the area most often affected, where border zone infarcts produce visual field defects (often partial and easily missed on routine examination), reading difficulties, visual disorientation and constructional apraxia. In the frontal border zone, slowing up, pathological grasp reflexes, gait disturbances and incontinence may occur.

It should now be apparent that the clinical description is helpful in formulating an option about the anatomical site, aetiology, prognosis and risk of recurrence. This may well have management consequences.

\section{TIMING/PATHOGENESIS}

The timing of events is important in our understanding of pathogenesis. Most TIAs are embolic. Embolic TIAs usually arise from the internal carotid artery or from the heart. A small percentage are haemodynamic - these usually occur when there is severe widespread occlusive disease.

Stroke is usually secondary to thromboembolic disease. About $15 \%$ of all strokes are haemorrhagic. Five per cent of these are secondary to subarachnoid haemorrhage and $10 \%$ to intracerebral haemorrhage (see box 3 ). Thromboembolic stroke accounts for $85 \%$ of all stroke. ${ }^{3}$ It is usually possible on clinical grounds to differentiate those with subarachnoid haemorrhage. The management of subarachnoid haemorrhage is beyond the scope of this review but is discussed elsewhere. ${ }^{4}$

The clinical differentiation of thromboembolic disease and intracerebral haemorrhage is difficult. Various authors have attempted to develop a clinical score. Early loss of consciousness, early vomiting, bilateral extensor plantars, marked elevation of blood pressure, all suggest haemorrhage. TIAs and the presence of peripheral vascular disease suggest thromboembolic disease. However, no clinical score is sufficiently accurate to allow reliable differentiation. Computer tomography (CT) scan (provided it is performed within two weeks of the first symptom) is the only reliable method; stroke patients should have a CT scan as clearly the proper assessment and treatment of thromboembolism and haemorrhage are different.

What percentage of strokes are thrombotic and embolic is even more difficult to ascertain. It is estimated that approximately half of all thromboembolic strokes are embolic (box 4) and half thrombotic. The percentage of all strokes due to other causes such as primary hypoperfusion ( $q v$ border zone infarcts), vasospasm and arteritis is small. 


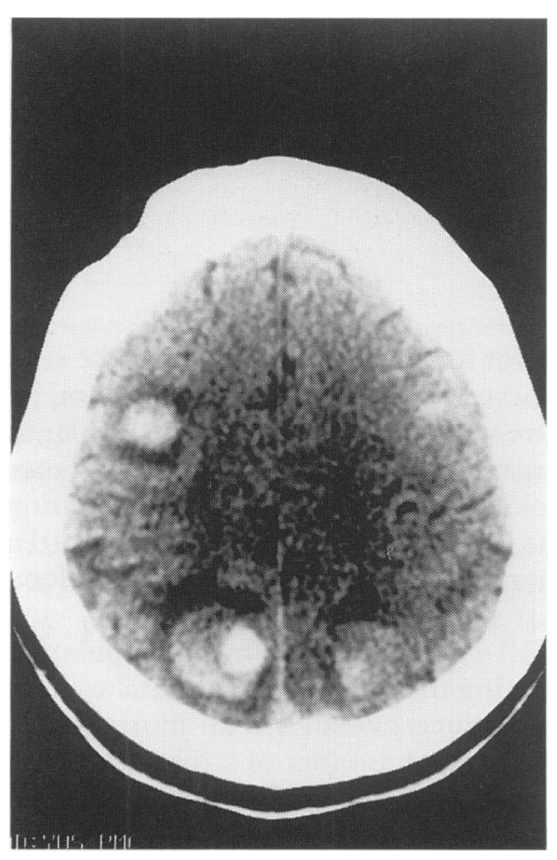

Figure 1 CT scan showing multiple intracerebral haemorrhages in a patient with cerebral amyloid

\begin{tabular}{|l|}
\hline Cardiac sources of emboli \\
\hline Left atrium \\
- thrombus (usually secondary to \\
atrial fibrillation) \\
- myxoma \\
- paradoxical embolism \\
Mitrial septal aneurysm \\
Mitral valve \\
- infeumatic endocarditis \\
- marantic endocarditis \\
- prosthetic valve \\
- mitral valve prolapse \\
Left ventricle \\
- thrombus - myocardial infarction, \\
cardiomyopathy \\
Aortic valve \\
- rheumatic endocarditis \\
- infective endocarditis \\
- marantic endocarditis \\
- aortic sclerosis and calcification \\
- prosthetic valve \\
- syphilitic aortitis \\
Congenital cardiac disorders \\
Cardiac surgery \\
- air embolism \\
platelet/fibrin embolism \\
\hline
\end{tabular}

Box 4

\section{Accuracy of diagnosis}

The differential diagnosis of TIA and stroke includes epilepsy, migraine, tumour, demyelination, syncope, subdural haematoma, malignant hypertension, . hyperventilation, hypoglycaemia and giant intracerebral aneurysm. ${ }^{5}$

Focal motor seizures may be mistaken for TIA, especially in patients with severe carotid stenosis in whom the jerking of the limbs occurs as part of the TIA. Focal sensory seizures are even more difficult to distinguish although the march of the sensory symptoms in a focal seizure may be helpful.

Migraine occasionally presents diagnostic difficulties. The slow build up of a migrainous aura which often lasts $20-30$ minutes would be unusual in a TIA. Visual migraine is often made up of positive visual symptoms, eg, scintillating scotomas, unlike the blackness of amaurosis fugax. The presence of a typical migrainous headache would be unusual in a TIA. Headache occurs in $16 \%$ of TIA patients.

The UK TIA study group have recently presented their data on tumours mimicking TIAs. Patients who present with sensory TIAs, jerking TIAs, loss of consciousness or speech arrest should all be suspected of having a tumour until proven otherwise. ${ }^{6}$

Demyelination will usually be suspected on the basis of the age of the patient, past history of previous attacks and more gradual onset of hemiparesis compared to that seen in a vascular hemiparesis.

Subdural haematomas rarely present with vascular-like symptoms. They do, however, present a particular diagnostic difficulty. The diagnosis of a carotid TIA is usually reasonably consistent. However, vertebrobasilar TIAs are more variable. It is important to be wary of labelling the following as a TIA - loss of consciousness, dizziness, mental confusion, incontinence of faeces or urine and bilateral loss of vision with reduced level of consciousness - these are all often secondary to hypoperfusion, following primary cardiac disease. Isolated symptoms such as vertigo, diplopia, dysphagia, dysarthria, loss of balance, tinnitus, sensory symptoms confined to one part of one limb or face, amnesia, drop attacks and scintillating scotomas, should always be interpreted cautiously when they occur in isolation: they may, however, be consistent with TIAs especially if they occur together or with other more definite symptoms. It is of particular concern to see a survey funded by the Stroke Association showing that, in the community, TIAs are thought to be diagnosed 10 times more frequently than they actually occur (S Pollock, personal communication). I spend a lot of time 'undiagnosing' TIAs.

In the diagnosis of stroke the false positive rate with no investigations is between 1 and $5 \%$ if a careful history is taken of the event. It is important to emphasize that CT is no more accurate than the clinical opinion; this is probably because some events which are clinically strokes are mistakenly diagnosed on CT as tumours, a diagnosis which is not substantiated with time.

\section{Risk factors}

Age is the most important risk factor for stroke. Hypertension is the most important treatable risk factor. ${ }^{7}$ The risk of stroke after a TIA is $30 \%$ in five years, the highest risk being in the first year. ${ }^{8}$ Other proven risk factors include cardiac disease, diabetes mellitus, smoking ${ }^{9}$ and hypercholesterolaemia. ${ }^{10}$ Cholesterol is also a major risk factor for heart disease which will be the cause of death in most patients with cerebrovascular disease.

Alcohol, in excess, is probably a risk factor for cerebrovascular disease, especially haemorrhage. Raised homocysteine and fibrinogen levels may be independent risk factors for vascular disease. ${ }^{11,12}$

It is not known if obesity, stress, or physical activity have any part to play in the aetiology of stroke - if they do, it is likely to be small.

\section{Investigations}

Few basic investigations are necessary for most patients with TIAs. All should have a full blood count, erythrocyte sedimentation rate, urea and electrolytes, glucose and cholesterol. Many physicians request a chest X-ray and electrocardiogram (ECG), although whether these are necessary is debatable if there are no symptoms or signs of cardiac disease.

Carotid TIAs or stroke with recovery also need a Doppler/duplex ultrasound to detect carotid stenosis - this is highly accurate but very operator dependent; ${ }^{13-15}$ our own experience has shown that most X-ray departments setting up Doppler/duplex ultrasound services are highly inaccurate and all such units 


$$
\begin{aligned}
& \text { Investigations for patients } \\
& \text { with TIAs } \\
& \text { - thyroid functions tests - especially } \\
& \text { when cholesterol is raised or patient } \\
& \text { is in atrial fibrillation } \\
& \text { - CT scan is indicated if there is:- } \\
& \text { - doubt about diagnosis, especially } \\
& \text { if gradual onset or no clear history } \\
& \text { - need to exclude cerebral } \\
& \text { haemorrhage - ideally all strokes } \\
& \text { - cerebellar stroke with } \\
& \text { deteriorating level of } \\
& \text { consciousness } \\
& \text { - cerebral angiography (digital } \\
& \text { subtraction/MRI) may be indicated } \\
& \text { if the patient is a candidate for } \\
& \text { carotid endarterectomy } \\
& \text { - to look for evidence of arteritis } \\
& \text { - in cerebral haemorrhage } \\
& \text { echocardiography is indicated if:- } \\
& \text { - abnormal cardiac findings are } \\
& \text { present suggestive of valvular } \\
& \text { heart disease or evidence of recent } \\
& \text { myocardial infarction or left } \\
& \text { ventricular aneurysm clinically or } \\
& \text { on chest X-ray or ECG } \\
& \text { - all young stroke (<50 years) - if } \\
& \text { no other clear cut cause is found } \\
& \text { - multiple territory stroke for no } \\
& \text { clear cause } \\
& \text { - antiphosphospholipid syndrome } \\
& \text { or systemic lupus erythematosus } \\
& \text { ?Liebman Sachs endocarditis } \\
& \text { - positive blood cultures } \\
& \text { - blood cultures - if febrile - } \\
& \text { subacute bacterial endocarditis } \\
& \text { temporal artery biopsy - arteritis } \\
& \text { - } 24 \text { h ECG - arrhythmia (very rarely } \\
& \text { necessary: grossly over used) } \\
& \text { - young stroke work up } \\
& \text { ond }
\end{aligned}
$$

should have their results substantiated either by angiography or a proven ultrasound service.

Other tests to be considered are included in box 5 .

\section{Treatment}

MEDICAL

\section{Vascular risk factors}

Hypertension is the most important risk factor for stroke. The risk of stroke rises exponentially as diastolic blood pressure increases in the range $70-100 \mathrm{mmHg}$. A $7.5 \mathrm{mmHg}$ rise in diastolic blood pressure within the range $70-110 \mathrm{mmHg}$ is associated with a doubling in the risk of stroke. This emphasizes the importance of blood pressure control including in the elderly. There is a risk of precipitating hypotension in a small number of patients, especially the elderly; this is often overstated as a reason for not being more aggressive about treating blood pressure, especially isolated systolic hypertension.

In the population at large a modest fall of $5 \mathrm{mmHg}$ in mean population diastolic blood pressure, achievable by reducing the mean daily salt intake in the population by a modest $50 \mathrm{mmol} / 1 \mathrm{might}$ reduce overall stroke mortality by $22 \%{ }^{16}$ This would have a greater effect on the total number of strokes than just treating high blood pressure in people with diastolics of over $100 \mathrm{mmHg}$. Treating all hypertensives would reduce the mortality of stroke by $15 \%$. This compares to aspirin which reduces the overall incidence of stroke by $1-2 \%$ and carotid endarterectomy which reduces the overall incidence by $0.5 \%{ }^{17}$ Recent data suggest that inadequate treatment of high blood pressure is common and is the most important avoidable risk factor which is not being adequately monitored and treated. ${ }^{18}$ These figures emphasize that treating blood pressure is crucial and will do more for stroke than any other treatment, both surgical and medical.

Advice about smoking is clearly important. Good diabetic control is to be encouraged. There is no consensus about the value of cholesterol-lowering drugs. There is, however, no doubt that the lower the cholesterol the lower the chance of heart attack. The value of lipid-lowering drugs in TIA and stroke patients is under investigation.

A small number of patients have haematocrits over 50 and these need assessment for polycythaemia followed by appropriate treatment, if necessary.

\section{Acute stroke}

In recent years there has been much debate over the value of stroke units. A recent overview leaves little doubt that patients treated in stroke units do better than those treated in general medical wards. ${ }^{19}$ There is no specific reason for this: it may just be an organisational matter rather than due to any one specific treatment.

More difficult is the question of whether patients do better treated at home or in hospital. The recent papers from Gladman $e t$ al ${ }^{20}$ and Young and Forster, ${ }^{21}$ have suggested home may be better. More work needs to be done on this. If the patient is kept at home, it is clearly important that he should still be investigated in the most appropriate manner. What is needed now are more trials comparing home with stroke units.

High blood pressure after acute stroke is common and often settles spontaneously. In most people it does not require treatment. One should only start treatment if hypertensive encephalopathy is considered possible (systolic $>230 \mathrm{mmHg}$ : diastolic $>130 \mathrm{mmHg}$ ) or the patient has had a proven cerebral haemorrhage and the blood pressure is markedly elevated. It is also important to check the blood pressure in all strokes 1-2 months after discharge from hospital as a significant number will show a persisent rise in blood pressure after discharge which is severe enough to require treatment, even though that individual's blood pressure will have been satisfactory at the time of hospital discharge.

There is no proven medical treatment for acute stroke. Dextran has been shown not to be beneficial. The trials of calcium antagonists, steroids and glycerol are inconclusive. ${ }^{22}$ This is an area of active research.

The International Stroke Trial (IST) is comparing heparin, aspirin and placebo. The Multi-centre Acute Stroke Trial (MAST) is assessing thrombolysis. This whole area has recently been reviewed. ${ }^{23}$ In spite of the lack of any proven treatment it is one of the most exciting frontiers in acute neurology. There is no doubt that treatment will come: the increase in and interest created by large multi-centre trials is rightly unstoppable. Stroke units make these trials much easier to perform. We have only to see how the ISIS trials have 
transformed the care of myocardial infarction to know that we must encourage stroke units to investigate treatments for this most debilitating disease.

\section{TIAs/stroke with recovery}

Aspirin reduces the risk of stroke and death in patients with TIAs but by only $25 \%{ }^{24}$ The exact dose is unclear. The evidence is best for doses of around $300 \mathrm{mg}$. Some believe smaller doses $(37.5 / 75 \mathrm{mg}$ ) may be adequate but it is possible that the trials using these doses may be too small and that we may have missed a difference between $37.5 \mathrm{mg}$ and larger doses due to a Type II error.

Ticlopidine is also an effective antiplatelet agent which may be more effective than aspirin. ${ }^{25}$ It is available in the US but not on general release in the UK. Unfortunately it sometimes causes skin rash, diarrhoea and reversible neutropenia - patients, therefore, need more careful monitoring. A sister to ticlopidine - clopidogrel - is at present undergoing trials comparing it with aspirin.

Warfarin is indicated for definite cardiac emboli. Lone atrial fibrillation has now been added to this list. The European atrial fibrillation study shows that warfarin reduces the risk of subsequent stroke by $60-70 \%$ compared to placebo in patients who have had an episode of cerebral ischaemia. The risk of serious bleeding was only $3 \%$ per annum with $0.2 \%$ intracranial bleeds. ${ }^{26}$

I also use warfarin if someone has had several TIAs not controlled by aspirin. I tend to give warfarin for 6-12 months and then switch back to aspirin provided no further ischaemic events occur. Trials are being performed comparing aspirin with warfarin.

A common question is when to start warfarin after a definite stroke. The risk of recurrent emboli is high after the first event but there is the danger of secondary haemorrhage into an infarct if warfarin/heparin is started too early. The Cardiac Embolism Study group have shown that the risk of secondary haemorrhage is very low by 11 days after the initial event. The risk is also very low in the first 11 days if the infarct is small or the deficit mild. Therefore, I start anticoagulants immediately if the defect is mild but delay for 11 days if the deficit is severe (eg, severe hemiparesis, sensory and dysphasia with a large infarct on CT: clearly on day one CT may be negative and the decision then has to be made on the severity of the clinical deficit only). ${ }^{27}$

\section{SURGICAL TREATMENT}

In 1954 the first carotid endarterectomy was performed. The risk of stroke in patients with a carotid stenosis who have had a TIA is approximately $10 \%$ per annum. The quoted surgical risk of carotid endarterectomy varies from $1 \%$ to $25 \%$. It is not surprising, therefore, that for 37 years no one knew if this operation was worthwhile. It is only with the publication of the European Carotid Surgery Trial (ECST) and the North American Trial (NASCET) in 1991 that the true value of this surgery became known. ${ }^{28}$

The serious complication rate in ECST was 3.7\% and in NASCET $2.1 \%$. Patients were randomised to surgery and medical treatment or best medical treatment. In the group with $70-99 \%$ stenosis, there was a highly significant benefit from surgery; there were $75 \%$ fewer strokes in those treated with carotid endarterectomy. Clearly, the lower the surgical complication rate the sooner the patient benefits from surgery. In the ECST trial the crossover was at approximately five months and in NASCET three months. All fit patients with a tight symptomatic stenosis should, therefore, be offered surgery (box 6); they should be given the natural history risks of stroke (ie, $10 \%$ per annum), the local operative risk and advised that surgery reduces the risk of stroke to $2-3 \%$ per annum. Each patient can then make up his/her mind whether they wish to pursue this. If the surgical risk is over $10 \%$ then the benefit from this operation is lost: units should aim for a surgical risk of under $5 \%$.

There are a possible 5000 candidates for carotid endarterectomy in England and Wales: surgery to this group would prevent 500 strokes in the first year. It is not a cheap form of treatment but for the individual with a carotid TIA and a tight stenosis, surgery reduces the risk of a stroke by $75 \%$. The average District General Hospital of 250000 would expect to have approximately 20 patients who are fit, symptomatic and have a carotid stenosis of $>70 \%$. The value of this operation is crucially dependent on low operative mortality and morbidity.

It is important to appreciate that this operation is only for patients with recent carotid symptoms - ie, usually amaurosis fugax, hemiparesis, hemisensory loss and dysphasia. Just how much this operation has been overused is emphasized by the report in 1988 (before the results of the ESCT and NASCET trials) which showed that only $35 \%$ of patients had this operation for appropriate reasons in a sample of 1302 patients in the USA. ${ }^{29}$ 


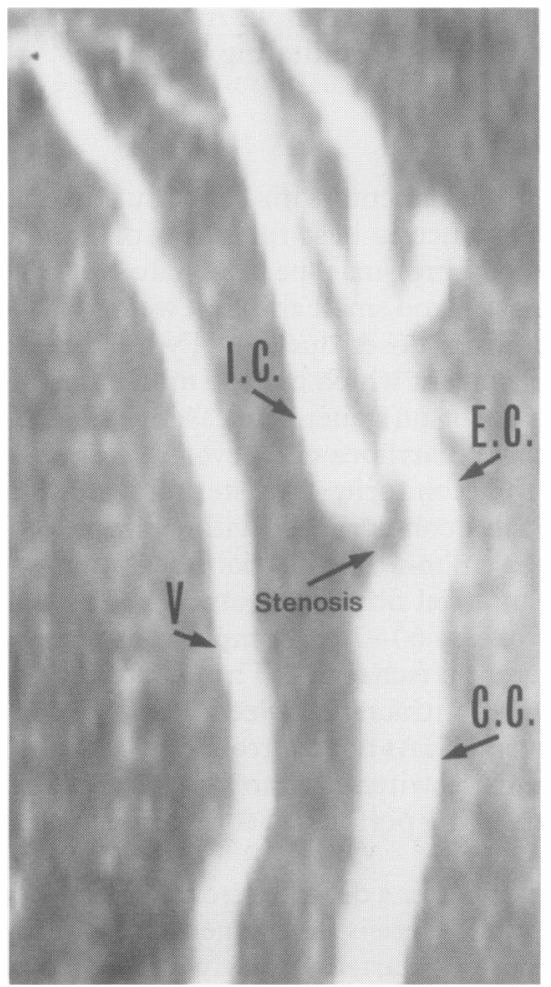

Figure 2 MRA showing a tight internal carotid stenosis. With tight stenoses, a 'gap' is quite frequently seen: in our experience, the presence of a 'gap' always means the stenosis is more than $80 \%$. (C.C.: common carotid; I.C.: internal carotid; E.C.: external carotid; V: vertebral)
Ultrasonography/angiography

None of these surgical trials included the angiographic risk. While the risk of stroke after carotid angiography is generally quoted as $1 \%,{ }^{30}$ it is almost certainly higher in patients with carotid stenosis, leaving approximately $2 \%$ with a permanent disability. ${ }^{31}$ Doppler/duplex ultrasound is undoubtedly the best screening test but it is very operator dependent. Some units operate on ultrasound alone; unfortunately, obtaining information on the intracranial circulation is difficult with ultrasound. Ultrasound combined with magnetic resonance angiography (MRA) gives highly accurate information about both the carotid artery in the neck as well as an angiographic picture of the whole intracerebral circulation.

Our policy now is to operate on the basis of an entirely non-invasive work up with ultrasound and MRA if both tests agree..$^{32}$ In our hands in the $70-99 \%$ stenosis group the tests agree in $96 \%$ of cases: we only pursue digital subtraction angiography on the $4 \%$ in whom the non-invasive tests do not agree. If MRA proves to be less operator dependent and becomes widely available it may replace the need for ultrasound; to do this would require an enormous expansion in MRA to all district general hospitals.

In expert hands a bruit is the best clinical guide to detect an underlying internal carotid stenosis. However, the bruit is lost in very tight stenoses (false negative); a false positive bruit is also not infrequent in the presence of either a contralateral occlusion, external carotid stenosis, or just internal carotid atheroma. Furthermore, if the presence of a bruit is to be of value, it needs to be useful to those who are making the initial assessment. The presence or absence of a bruit mentioned in the referral letter to a cerebrovascular clinic showed a specificity of $70 \%$ and sensitivity of $57 \%$ for $70-99 \%$ stenosis. ${ }^{33}$ On this basis many patients with a carotid stenosis would be denied surgery if only those with a bruit were referred. All patients with carotid TIA or stroke with recovery should have carotid ultrasound in a department with a proven track record. I no longer listen for a bruit; if I wish to detect a carotid stenosis I perform an ultrasound examination.

My own personal work-up for carotid endarterectomy is, therefore, a careful history, simple examination of the cardiovascular system (occasionally I examine the neurological system), routine blood tests, chest X-ray and ECG. This combined with a Doppler/duplex ultrasound is all done at the first clinic visit. If the patient is found to have a carotid stenosis and is prepared to take the risk of surgery, then an urgent MRA is booked as an out-patient.

I do not routinely perform CT or magnetic resonance imaging (MRI) scans on these patients. Tumour 'TIAs' are rare and can often be suspected on clinical grounds - patients with speech arrest, pure sensory TIA, blackouts, and jerking in their TIAs should all raise the suspicion of alternative pathology. CT need only be performed in this group.

\section{Assesment for surgery}

I remain convinced that a neurologist or physician with a major interest in vascular disease should perform the initial assessment. These are not patients who should be referred primarily to the vascular surgeons. In our cerebrovascular clinics, we see 25 new patients each week, only $2-3$, on average, meet all the criteria to be candidates for carotid endarterectomy. The differential diagnoses seen in our clinic include migraine, epilepsy, hyperventilation, tumours, Parkinson's disease and motor neurone disease, to name but a few. In a population of a million people, there are approximately 50-100 people who are candidates for carotid endarterectomy each year: this compares with approximately 5000 people with asymptomatic carotid stenosis. The scope for inappropriate surgery is substantial. It is unreasonable to expect a vascular surgeon to perform this filtering.

There is little doubt that the risk of a stroke is highest in the first six months after the initial event and if the time for first symptom to assessment, investigation and surgery, takes several months, then we are missing the boat in significant numbers of patients. In the UK we need to assess these patients within a few days of their symptoms and prepare patients for surgery if appropriate within two weeks after TIAs and 6-8 weeks after strokes with recovery. This will require changes in organisation, more neurologists with an interest in vascular disease and perhaps more vascular surgeons.

Hydrocephalus

Hydrocephalus is a complication of cerebellar strokes (both haemorrhage and infarction) which may be amenable to surgical treatment. 
Asymptomatic bruits

These are common in the elderly population (approximately $7 \%$ over age 65 ). The risk of ipsilateral stroke is approximately $2 \%$ per annum. Few would advocate surgery.

\section{OTHER ASPECTS}

\section{Emotional aspects}

These are very important; few doctors have sufficient time to address these fully. Depression and anxiety are common: with reassurance, especially about the risk of recurrence and advice about treatment to prevent further events, this often improves with time. Counselling the spouse and close family is also important.

Emotionalism is also common: it is present in both bilateral and unilateral strokes. Often it is sufficient to explain this is a physical symptom which improves with time: sometimes small doses of amitriptyline $(10-25 \mathrm{mg})$ may be highly beneficial.

\section{Epilepsy}

Early epilepsy occurs in approximately $10 \%$ of all strokes; it should be energetically treated (eg, with intravenous phenytoin) as the cerebral metabolic rate doubles during a fit. Late epilepsy after a stroke is a common cause of epilepsy in the elderly population in general; however, it is rare in the individual stroke patient and should also make one reassess the validity of the diagnosis of cerebrovascular disease.

\section{Dysphagia}

This is common even after unilateral strokes. It usually recovers but predisposes to aspiration, chest infection, dehydration and death. It can be assessed by simply asking the patient to drink $50 \mathrm{ml}$ of water and should be mandatory in all stroke patients.

\section{Thalamic pain}

This is more common that is generally appreciated; it frequently starts weeks after the patient is discharged from hospital. It is a cause of great distress and may be helped by a variety of strategies. ${ }^{34}$

Other common problems

After acute stroke, deep vein thrombosis occurs in more than $50 \%$ of paretic legs, although a relatively small number develop symptomatic pulmonary embolism. Deep vein thrombosis can usually be managed with elasticated stockings. Pressure sores, septicaemia often secondary to urinary tract or chest infection, and hyperglycaemia should be watched for. Frozen shoulder is a common problem and can markedly slow the individual's recovery.

\section{Prognosis}

\section{Learning/summary points}

- treatment of high blood pressure is the most important factor in stroke prevention

- stroke patients are more likely to be alive and independent at six months if managed in stroke units rather than in medical wards

- aspirin reduces the risk of stroke by only $25 \%$. Carotid endarterectomy reduces the risk by $75 \%$

- dizziness and loss of consciousness are usually not TIAs. Hypotension is a much more likely cause

- only consider carotid endarterectomy if there are carotid symptoms and more than $70 \%$ stenosis. The operative risk of stroke during surgery must be under $10 \%$ (and preferably under $5 \%$
The risk of a stroke after TIAs is approximately $30 \%$ in five years. It is highest in the first year: in patients with carotid stenosis it is approximately $10-12^{\circ}{ }_{0}$ in the first year after the TIA. The figures for stroke are similar: it is, therefore, important to reassure all stroke patients that the second stroke is not just around the corner. Even patients with bilateral internal carotid occlusions only have a recurrent stroke risk of $13 \%$ per year.

Death in most patients with TIAs and stroke is due to cardiac disease. Following an acute stroke $20-30 \%$ of patients die. A poor prognosis is associated with reduced consciousness, conjugate gaze palsy, signs of severe brainstem dysfunction, pupillary changes and incontinence persisting beyond the first few days. Strokes resulting in cognitive impairment (eg, apraxia, neglect) and visuospatial dysfunction also carry a poor prognosis for recovery.

One year after a stroke, $33 \%$ of patients will be dead, $22^{\circ}{ }_{\mathrm{o}}$ dependent and $45^{\circ} \mathrm{o}$ independent. Most recovery occurs in the first few weeks: less recovery occurs in months 3-6 and even less, but still useful recovery, occurs in months $6-12$. It is known that some symptoms can show recovery over a long period of time, eg, hemiplegic leg, while others often do not recover much unless they recover early, eg, retinal infarction, homonymous hemianopia and isolated spinothalamic sensory loss. For the hemiplegic hand, if there is no active hand grip by three weeks there is unlikely to be useful recovery. It is crucial to take the natural history of disability into account when planning rehabilitation. 


\section{Young stroke}

Young stroke is often not premature atheroma. ${ }^{35}$ It should be investigated by a neurologist. The most common causes are emboli from the heart, carotid/ vertebral dissection, antiphospholipid syndrome, arteritis, cerebral venous thrombosis and premature atherosclerotic/hypertensive vascular disease. These require active assessment and treatment. It is likely that cerebral venous thrombosis should be treated with anticoagulation.

\section{Concluding remarks}

Delivering stroke care is expensive. Much progress has been made in recent years especially regarding the use of aspirin, warfarin and carotid endarterectomy. Large multi-centre trials should provide more data on the treatment of acute stroke and prevention of stroke over the next 5-10 years. This is one of the most exciting therapeutic frontiers in medicine.

1 Oxfordshire Community Stroke Project. Incidence of stroke in Oxfordshire. First year's experience of a Community Stroke Project. experience of a Comm 1983; 287: 713-6.

2 Fisher CM. Lacunar strokes and infarcts: a review. Neurology 1982; 32: 871-6.

3 Sandercock P, Molyneux A, Warlow CP. Value of computed tomography in patients with stroke: Oxfordshire Community Stroke Project. BMF 1985; 290: 193-7.

4 Van Gijn J. Subarachnoid haemorrhage. Lancet 1992; 339: 653-5.

5 Norris JW, Hachinski VC. Misdiagnosis of stroke. Lancet 1982; i: 328-331.

6 Coleman RJ, Bamford JM, Warlow CP. For the UK TIA Study Group. Intracranial tumours that mimic transient cerebral ischaemia: lessons from a large multicentre trial. $\mathcal{f}$ Neurol from a large multicentre trial. $\mathcal{F}$

7 Kannel WB, Wolf PA. Epidemiology of cerebKannel WB, Wolf PA. Epidemiology of cerebrovascular disease. In: Ross Russell, ed: Vascular disease of the central nervous system, Edin-
burgh: Churchill Livingstone, 1983; pp 1-24. 8 burgh: Churchill Livingstone, 1983; pp 1-24. 8 Dennis M, Bamford J, Sandercock P, Warlow C. Oxfordshire Community Stroke Project. Stroke 1990; 21: 848-53.

9 Donnan GA, McNeil JJ, Adena MA, Doyle AE, O'Malley HM, Neill GC. Smoking as a risk
factor for cerebral ischaemia. Lancet 1989; iii: 643-7.

10 Qizilbash N, Duffy SW, Warlow CP, Mann J. Lipids are risk factors for ischaemic stroke, overview and review. Cerebrovasc Dis 1992; 2: overview

11 Clarke R, Daly L, Robinson K, et al. Hyperhomocysteinaemia: an independent risk factor for vascular disease. $N$ Engl f Med 1991; 324: $1149-55$.

12 Qizilbash N, Jones L, Warlow CP, Mann J. Fibrinogen and lipid concentrations as risk factors for transient ischaemic attacks and minor ischaemic strokes. BMF 1991; 303: 605-9.

13 Humphrey P, Sandercock P, Slattery J. A simple method to improve the accuracy of non-invasive ultrasound in selecting TIA patients for cerebral angiography. $f$ Neurol Neurosurg Psychiatry 1990; 53: $966-71$.
14 Hankey GJ, Warlow CP. Symptomatic carotid ischaemic events: safest and most cost effective way of selecting patients for angiography, before way of selecting patients for angiography, before carotid $1485-91$

15 Howard G, Cambless LE, Baker WH, et al. A multicentre validation study of Doppler ultrasound versus angiography. $f$ Stroke Cerebrovasc Dis 1991; 1: 166-73.

16 Law M, Frost C, Wald N. By how much does dietary salt reduction lower blood pressure II. Analysis of data from trials of salt reduction. $B M \mathcal{F}$ 1991; 302: 819-24.

17 Dennis M, Warlow C. Strategy for stroke. BMF 1991; 303: 636-8.

18 Payne JN, Milner PC, Saul C, Bowns IR, Hannay DR, Ramsay LE. Local confidential inquiry into avoidable factors in deaths from inquiry into avoidable factors in deaths from 307: $1027-30$.

19 Dennis M, Langhorne P. So stroke units save lives: where do we go from here? $B M \mathcal{F}$ 1994; 309:
live lives: where.

20 Gladman JRF, Lincoln NB, Barer DH. A randomised controlled trial of domiciliary and hospital based rehabilitation for stroke patients after discharge from hospital. $f$ Neurol Neurosurg Psychiatry 1993; 56: 960-6.

21 Young JB, Forster A. The Bradford Community Stroke Trial, results at six months. BMF 1992; 304: 1085-9.

22 Sandercock $P$, Willems $H$. Medical treatment of acute ischaemic stroke. Lancet 1992; 339: 537-9.

23 Sandercock PAG, Van Der Belt AG, Lindley RJ, Slattery J. Antithrombotic therapy in acute RJ, Slattery J. Antithrombotic therapy in acute stroke: an overview of the completed randomized trials. $\mathcal{F}$

24 Antiplatelets Trialists Collaboration. Collaborative overview of randomised trials of Antiplatelet Therapy. Prevention of death, myocardial infarction and stroke by prolonged antiplatelet therapy in various categories of patients. BMF 1994; 308: 81-106.

25 Ticlopidine (Editorial) Lancet 1991; 337: 459-60.
26 European Atrial Fibrillation Study Group Secondary prevention in non rheumatic atrial fibrillation after transient ischaemic attack or minor stroke. Lancet 1993; 342: 1255-62.

27 Cerebral Embolism Study Group. Immediate anticoagulation of embolic stroke: brain anticoagulation of embolic stroke: brain 1984; 15: 779-89.

28 Brown MM, Humphrey PRD on behalf of the Association of British neurologists. Carotid endarterectomy: recommendations for management of transient ischaemic attack and ischaemic stroke. BMF 1992; 305: 1071-4.

29 Winslow CM, Solomon DH, Chassin MR, Kosecoff J, Merrick NJ, Brook RH. The appropriateness of carotid endarterectomy. $N \mathrm{Engl} f$ Med 1988; 318: 721-7.

30 Hankey GJ, Warlow CP, Sellar RJ. Cerebral angiographic risk in mild cerebrovascular angiographic risk in mild cer

31 Davies KN, Humphrey PRD. Complications of cerebral angiography in patients with symptomatic carotid territory ischaemia screened by carotid ultrasound. $f$ Neurol Neurosurg Psychiatry 1993; 56: 967-72.

32 Young G, Humphrey PRD, Shaw MDM Nixon TE, Smith ETS. Comparison of magnetic resonance angiography, duplex ultrasound, and digital subtraction angiography in assessment of extracranial internal carotid arter stenosis. $\mathcal{F}$ Neurol Neurosurg Psychiatry 1995; 57: $1466-78$.

33 Davies KN, Humphrey PRD. Do carotid bruits predict disease of the internal carotid artery? Postgrad Med f 1994; 70: 433-5.

34 Bowsher D. Pain syndromes and their treatment. Curr Opin Neurol Neurosurg 1993; 6 257-63.

35 Caplan LR. Stroke: a clinical approach. Butterworth Heinemann, 1993; pp 469-85. 